\title{
Clinically Mimicking Pancreatic and Dilation in Main Pancreatic Duct in Autoimmune Pancreatitis (AIP): A Case Report and Literature Review
}

\author{
Yaqin Li*, Xiaojia Xiong \\ Perking University of Shenzhen Hospital, Shenzhen, China \\ Email: ^1952459709@qq.com
}

How to cite this paper: Li, Y.Q. and Xiong, X.J. (2017) Clinically Mimicking Pancreatic and Dilation in Main Pancreatic Duct in Autoimmune Pancreatitis (AIP): A Case Report and Literature Review. Open Access Library Journal, 4: e3065.

https://doi.org/10.4236/oalib.1103065

Received: April 1, 2017

Accepted: May 12, 2017

Published: May 15, 2017

Copyright $\odot 2017$ by authors and Open Access Library Inc.

This work is licensed under the Creative Commons Attribution International License (CC BY 4.0).

http://creativecommons.org/licenses/by/4.0/ (c) (i) Open Access

\begin{abstract}
Autoimmune pancreatitis (AIP) is a unique form of pancreatitis which has autoimmune features. AIP has clinical presentations that greatly mimic pancreatic cancer (PC). It is critical for clinicians to distinguish AIP from PC because their treatments and prognoses are entirely different. We report a 62year-old man with obstructive jaundice and dilation in the main pancreatic duct (MPD). The diagnosis of AIP was based on diagnostic criteria by the elevated IgG-4 serum levels and the typical imaging such as diffuse pancreatic swelling and dilation in the common biliary duct (CBD). The difference of the imaging finding in our case is the expanding of the MPD, which is usually described to be stenosis in many literatures. Finally, the patient was confirmed with type I AIP according to the diagnosis criteria. After the steroid treatment lasting 3 months, the patient was gradually recovered. We present the case and take a review of literature in order to improve the early identification of AIP.
\end{abstract}

\section{Subject Areas}

Internal Medicine

\section{Keywords}

Autoimmune Pancreatitis (AIP), Pancreatic Cancer, Dilation in the MPD

\section{Introduction}

AIP is a rare cause of chronic pancreatitis, characterized by a fibroinflammatory process by lymphoplasmacytic infiltrates, storiform fibrosis, obliterative phlebi- 
tis, and increased IgG4+ plasma cells, leading to dysfunction of the pancreas. AIP was first characterised in 1961 from a patient showing signs of pancreatic damage with associated autoimmunity [1]. This notion received no further attention in Japan until the mid 90's when Yoshida et al. published a case of a patient with chronic pancreatitis and impaired exocrine function with associated autoimmunity [2]. The term AIP was first used here. In 2002, the Japan Pancreas Society (JPS) proposed the world's first clinical diagnostic criteria for AIP (JPS 2002) [3], which was revised in 2006 (JPS 2006) [4]. Subsequently, the International Consensus Diagnostic Criteria (ICDC) was proposed in 2010 [5]. We report a case of a 62-year-old man with obstructive jaundice, mild upper abdominal pain and darkening urine. A computed tomography (CT)s can shown the localized mass-forming strongly suspected of PC.

\section{Case Presentation}

A 62-year-old man was admitted to our hospital complaining of jaundice, mild upper abdominal pain and darkening urine. Past medical history and the family history were negative. Physical examination revealed icteric sclera and skin, mild upper abdominal tenderness. The laboratory test data at admission showed that the serum levels of alanine transaminase (ALT), aspartate transaminase (AST), alkaline phosphatase (ALP), $\gamma$-glutamyltransferase $(\gamma$-GT), total bilirubin (TBil) and direct bilirubin (DBil) were elevated to $84.8 \mathrm{U} / \mathrm{L}$ (9 - $50 \mathrm{U} / \mathrm{L}), 67.1 \mathrm{U} / \mathrm{L}(15$ $40 \mathrm{U} / \mathrm{L}), 448.2 \mathrm{U} / \mathrm{L}(45-125 \mathrm{U} / \mathrm{L}), 459.5 \mathrm{U} / \mathrm{L}(10$ - $60 \mathrm{U} / \mathrm{L}), 151.7 \mu \mathrm{mol} / \mathrm{L}(5-21$ $\mu \mathrm{mol} / \mathrm{L})$ and $100.8 \mu \mathrm{mol} / \mathrm{L}(0-3.4 \mu \mathrm{mol} / \mathrm{L})$, respectively. Serum IgG was 27.47 $\mathrm{g} / \mathrm{L}(7-16 \mathrm{~g} / \mathrm{L})$. Test of tumor markers from the bile showed CA199 of 6147.70 $\mathrm{U} / \mathrm{ml}(0-37.0 \mathrm{U} / \mathrm{ml})$. Both computed tomography $(\mathrm{CT})$ and magnetic resonance image (MRI) revealed diffusive enlargement of the pancreas (Figure 1 and Figure 2). Other abnormalities included significant dilation of intra- and extra-hepatic bile duct, diffusive thickening of the gallbladder wall. The differences of the

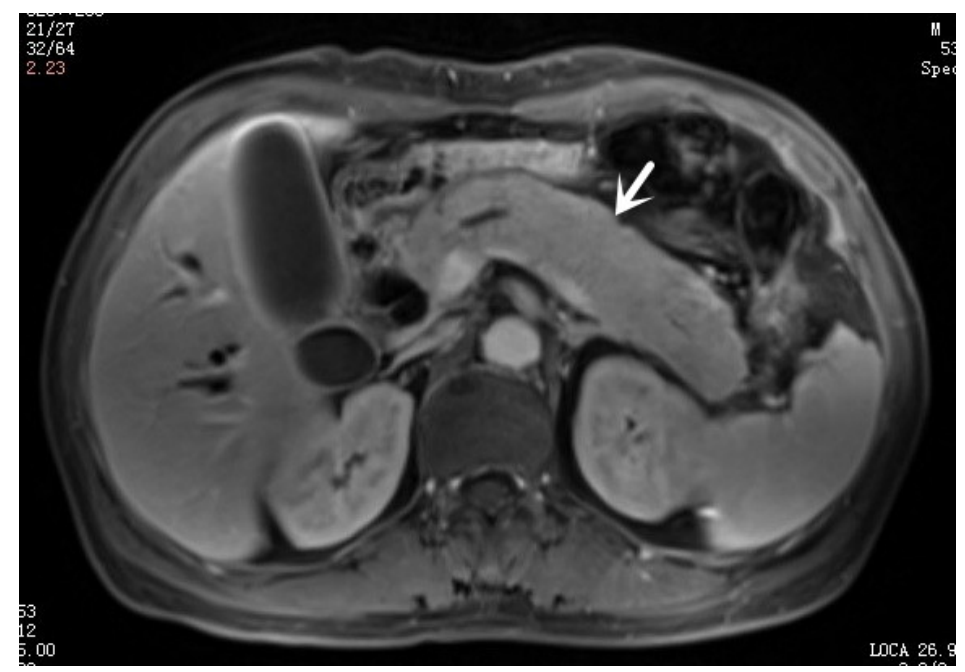

Figure 1. The sausage-like pancreas and expanding of the main MPD in MRCP. 


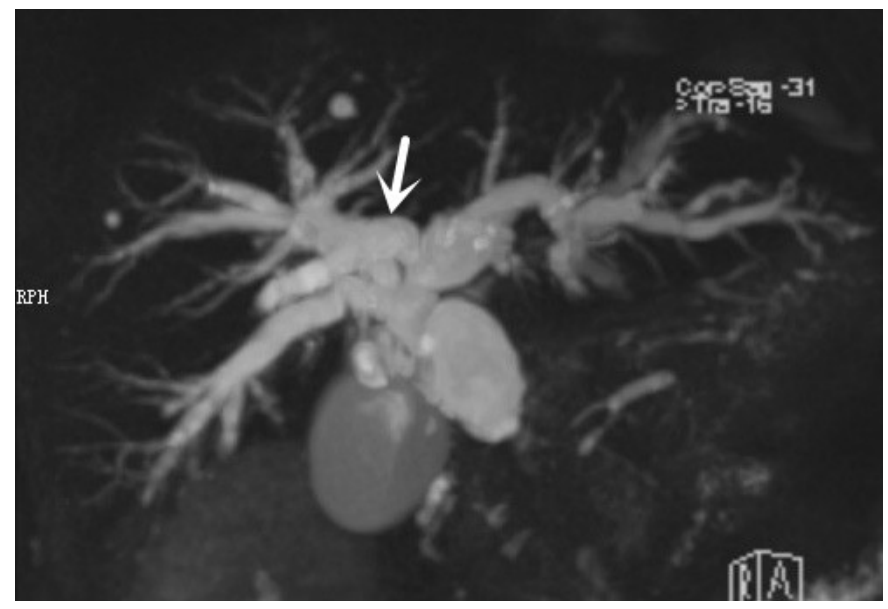

Figure 2. The dilation of intra- and extra-hepatic bile duct in MRCP.

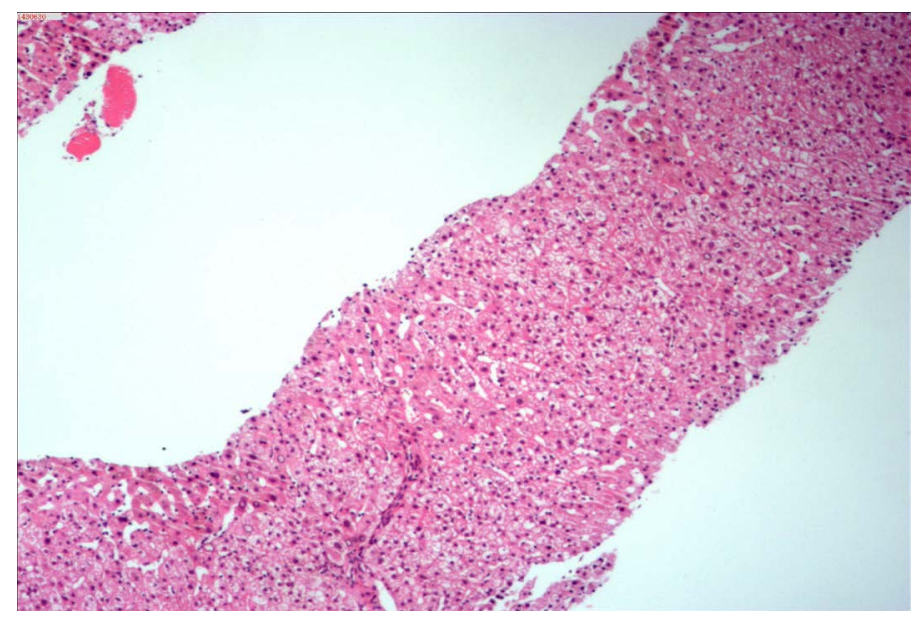

Figure 3. Warthin-Starry staining 40×.

imaging finding in our case is the expanding of the main pancreatic duct (MPD), which is usually described to be stenosis in many literatures. Endoscopic retrograde cholangio-pancreatography (ERCP) was conducted, revealing similar findings with CT and MRI. No sign of malignancy was observed. The liver biopsy was performed, the pathology manifestation revealed the inflammatory infiltration of the hepatocyte (Figures 3-6). The patient was suspected of suffering from AIP, the following examinations was conducted. IgG4 was measured and turned out to be $6.55 \mathrm{~g} / \mathrm{L}(0.03-2.01 \mathrm{~g} / \mathrm{L})$. So the diagnosis of type I AIP was confirmed in accordance with the diagnostic criteria [2]. Steroid therapy with oral prednisone (32.5 mg per day) was administered promptly. Serum ALP, $\gamma$-GP, TBil and DBil just before steroid therapy were $448.2 \mathrm{U} / \mathrm{L}, 459.5 \mathrm{U} / \mathrm{L}, 151.7$ $\mu \mathrm{mol} / \mathrm{L}$ and $100.8 \mu \mathrm{mol} / \mathrm{L}$, respectively, and one week later were $218.6 \mathrm{U} / \mathrm{L}, 128.3$ $\mathrm{U} / \mathrm{L}, 46.1 \mu \mathrm{mol} / \mathrm{L}$ and $19.7 \mu \mathrm{mol} / \mathrm{L}$, respectively. After six weeks, we stepped down the dosage ofprednisone with $27.5 \mathrm{mg}$ daily, and the laboratory values were improved persistently. After the treatment of 70 days, the serum levels of ALP, $\gamma$-GP, TBil, DBil, and IgG returned back into the normal range. The repeat 


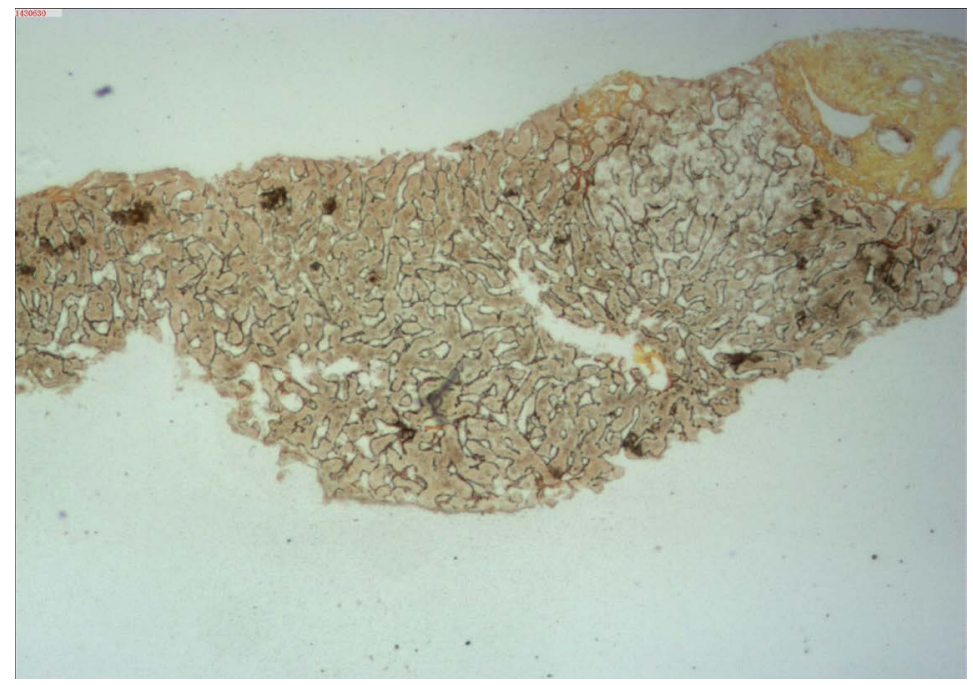

Figure 4. Masson staining 40×.

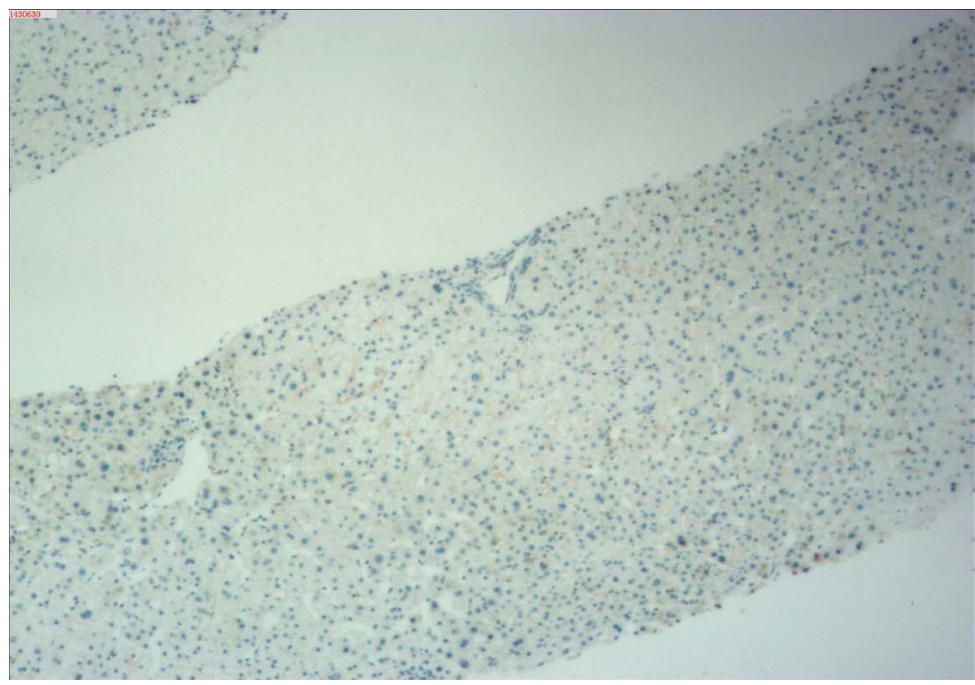

Figure 5. Warthin-Starry staining 40×.
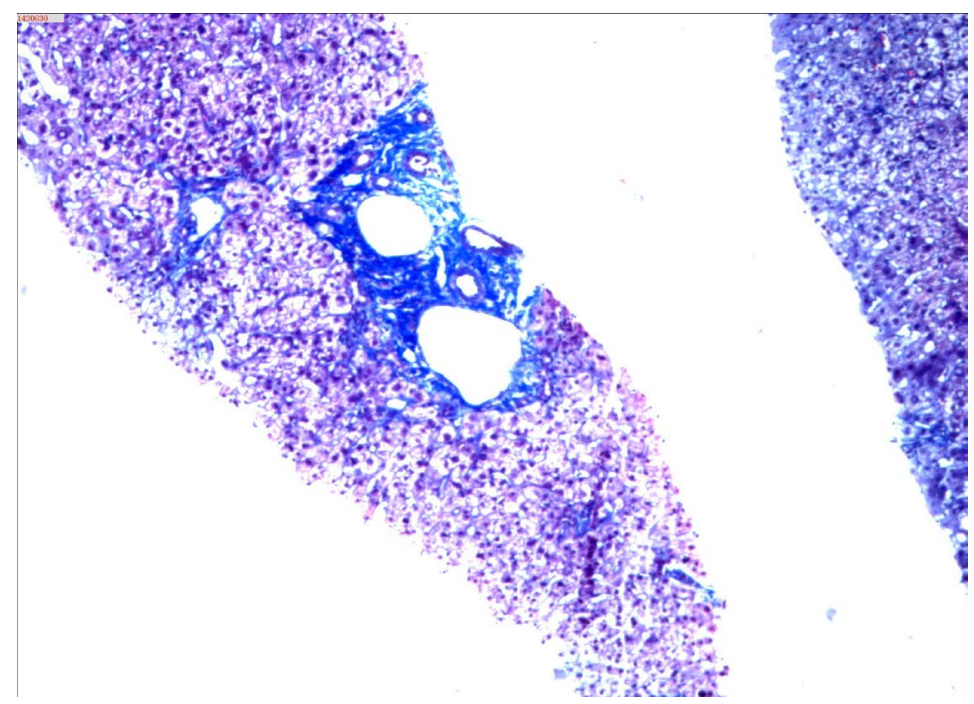

Figure 6. Masson staining 40×. 


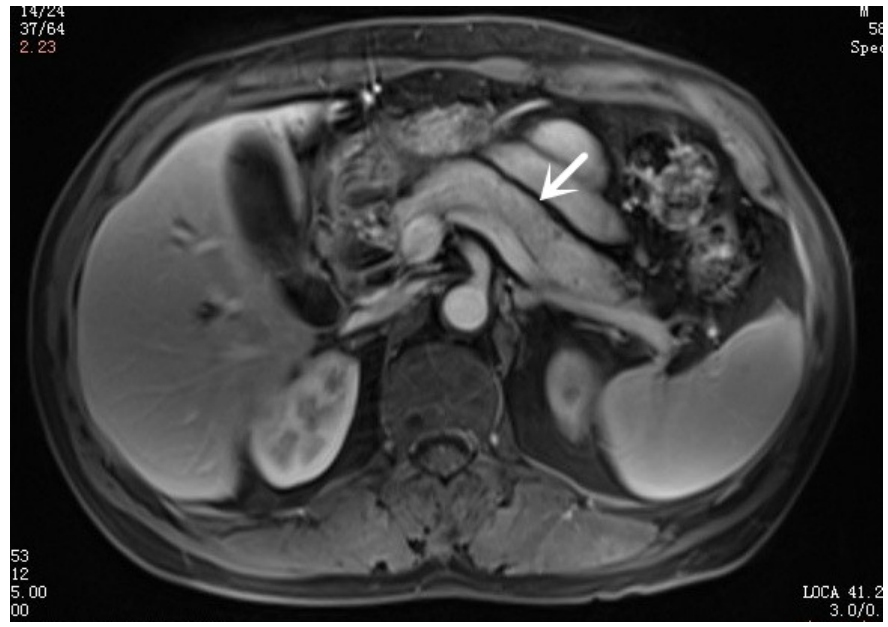

Figure 7. The swelling pancreas has remarkable reduced in MRCP.

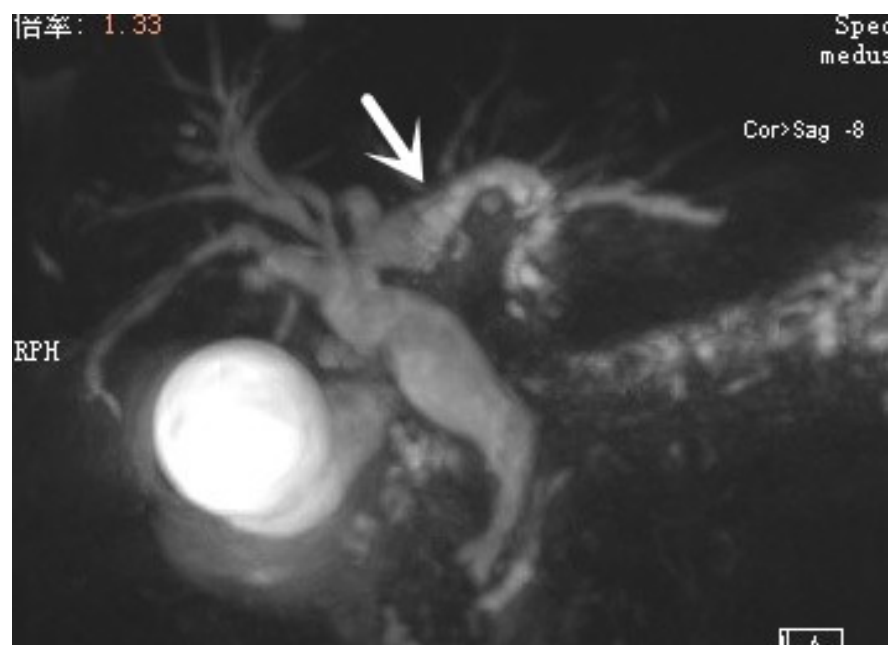

Figure 8. The enlargement of intra- and extra-hepatic bile duct has become small.

MRI showed remarkable reduction of the pancreas (Figure 7 and Figure 8). The patient was discharged and followed up at the clinic with prednisone tapered off every 2 weeks by $5 \mathrm{mg}$. The whole therapy has lasted 34 months, the patient are taking $5 \mathrm{mg}$ prednisone daily now. All the results of serology and imaging examinations are normal (Figure 9 and Figure 10).

\section{Conclusions}

\subsection{Classification of AIP}

Autoimmune pancreatitis (AIP) is a type of chronic pancreatitis occurring secondary to an autoimmune process. The international consensus diagnostic criteria (ICDC) for AIP recently described two subtypes: type 1 [lymphoplasmacytic sclerosing pancreatitis (LPSP)] and type 2 [idiopathic duct-centric pancreatitis (IDCP) or AIP with granulocytic epithelial lesion (GEL)] [5]. LPSP is the typical histopathological description of type 1 AIP. It is characterized by massive infiltration of lymphoplasmacytic cells without granulocytes; abundant ( $>10$ cells/ 


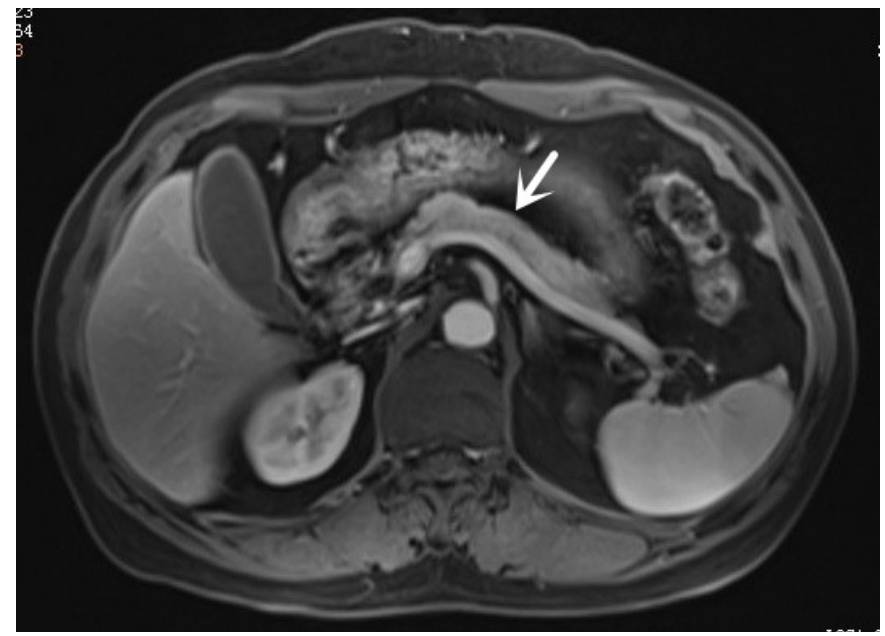

Figure 9. The normal imaging of pancreas.

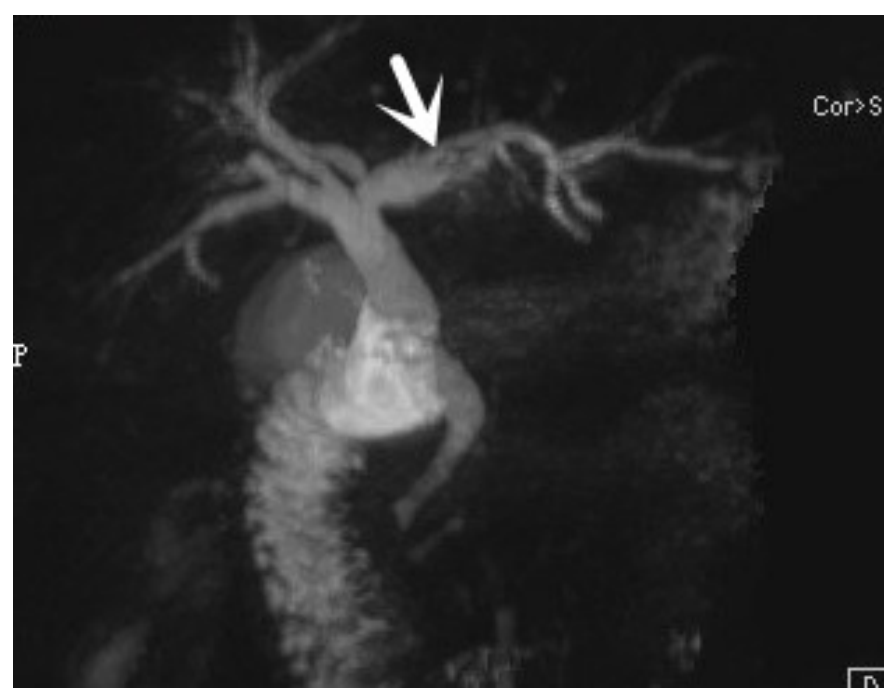

Figure 10. The intra- and extra-hepatic bile duct has almost become normal.

HPF) IgG4-positive plasma cells; storiform or swirling fibrosis and peri-venular infiltration with lymphoplasmacytic cells which often leads to obliterative phlebitis [6] [7] [8].

Type 2 AIP in contrast to type 1 AIP, is a pancreas-specific disease mostly without extra-pancreatic organ involvement, lacks elevated serum levels of IgG4 and auto antibodies. Type 2 AIP is associated with inflammatory bowel disease (approximately 30\%) and demonstrates little or no IgG4-positive inflammatory infiltrates on histology [9]. Histopathological review is required to confirm the diagnosis of type 2 AIP because of the lack of serological markers and specific imaging patterns.

\subsection{Clinical Presentation}

The clinical findings in AIP are non-specific and the most common presentation is obstructive jaundice. A recent systematic review of AIP in China showed that 
obstructive jaundice accounted for $63.4 \%$ of the 706 patients in the study [10]. This is fairly consistent with earlier findings of a multicenter survey of 731 patients, in which obstructive jaundice accounted for $75 \%$ and $47 \%$ of the patients with type 1 and type 2 AIP respectively [11]. In Asian patients the frequency goes from $74 \%$ to $88 \%$ [12] [13]. Abdominal pain was also reported with a higher frequency. Patients with type 2 AIP may present with severe abdominal pain (68\% vs. $41 \%$ ) in contrast to type 1 AIP patients. The abdominal pain in type 1 AIP is described as mild, not as severe as the abdominal pain observed in acute pancreatitis or acute exacerbation of chronic pancreatitis [5] [11] [14]. Extrapancreatic involvement has been mainly associated to LPSP with, in some cases, 2 to 4 organs affected [15]. The inflammatory bowel disease is the most frequent extrapancreatic manifestation. Other symptoms include back pain, weight loss and fatigue [14] [16].

\subsection{Serology}

The major serological finding is an elevation of the serum IgG4 concentration. The performance of total IgG4 serum concentrations for the diagnosis of AIP has been evaluated in different studies. A cut-off for IgG4 elevation was suggested at $\geq 135 \mathrm{mg} / \mathrm{dl}$ by Hamano et al. in 2001 with a sensitivity of $95 \%$ and a specificity of $97 \%$ [17]. The Mayo Clinic group reported a specificity and a sensitivity of $93 \%$ and $76 \%$, respectively, at a cut-off level of $>140 \mathrm{mg} / \mathrm{dl}$. In patients with IgG4 concentrations $>280 \mathrm{mg} / \mathrm{dl}$, a sensitivity of $53 \%$ and a specificity of $99 \%$ was found [18]. Although an elevated ratio of IgG4 to total IgG-positive plasma cells in tissue can confirm the diagnosis, approximately $30 \%$ of patients have normal values [5]. Other diseases such as atopic dermatitis, parasitic infections, pemphigus vulgaris, pemphigus foliaceus, and pancreatic carcinoma, can also be associated with elevated levels of IgG4 [19]. In spite of not disease-specific as highlighted above, IgG4 has the highest diagnostic value as a single serological test in AIP. The IgG4 serum concentration has been proposed as a noninvasive parameter for the diagnosis of AIP and is included in the HISORt criteria.

\subsection{Histology}

The traditional "gold standard" for the diagnosis of AIP is characteristic histology. The pancreatic lesion of type 1 AIP histologically shows LPSP with 3 essential features [20] [21]: 1) a lymphoplasmacytic infiltrate surrounding small-sized interlobular pancreatic ducts that does not destroy the pancreatic ductal epithelium; 2) a swirling fibrosis centered around ducts and veins (storiform fibrosis); and 3) obliterative phlebitis wherein the infiltrate surrounds and obliterates pancreatic veins. Destructive changes to the ducts and acini caused by infiltrating granulocytes are typically absent. Immunostaining reveals abundant IgG4positive cells ( $>10$ cells/HPF).

Type 2 AIP histology typically shows IDCP (AIP with GELs) [22], which is a distinct histological pattern from that of LPSP. The predominant interlobular stroma composed of lymphocytes plasma cells and reactive fibroblasts/myofi- 
broblasts seen in type 1 AIP is replaced by the presence of GELs as the most distinctive feature of IDCP. These changes may lead to the destruction and obliteration of the duct lumen, seen in the medium to small-sized ducts and also in the acini. Infiltrates of IgG4-positive plasma cells are scant or absent in IDCP [20] [21]. In Macarena study [23], the cystic lesion was intrapancreatic; it had a fibrotic wall without epithelial cells and contained a brownish fluid and some debris, resembling the usual findings in noninfected pseudocysts and not those in true neoplastic cystic lesions.

\subsection{Imaging}

Imaging plays an important role in the diagnostic work up for AIP as reflected in the different existing diagnostic criteria. AIP demonstrates a wide spectrum of imaging findings on CT. The characteristic CT appearance of autoimmune pancreatitis has been described as diffuse enlargement of the pancreas with a capsule-like rim. The pancreatic border becomes featureless with effacement of the lobular contour of the pancreas. There is diffusely decreased enhancement in the pancreas during the early phase and delayed enhancement in the late phase of contrast enhancement. Involvement of other organs, such as the biliary tree, retroperitoneum, salivary glands, and kidneys, is common [24].

MRI is a useful tool to evaluate pancreatic lesions. Contrast-enhanced fat suppression MRI has been studied to differentiate AIP and PC. Sugiyama shown that speckled enhancement within a hypointense or isointense lesion on pancreatic phase DCE-T1WI (speckled type) was observed more frequently in focal AIP than in PC, with high sensitivity, high specificity, and high accuracy [25].

Magnetic resonance cholangiopancreatography (MRCP) has become popular as a noninvasive method for obtaining high quality images of the pancreaticobiliary tree; MRCP is replacing diagnostic ERCP in many pancreatobiliary diseases. There has been a controversy in usefulness of MRCP in diagnosis of AIP. The major problem with MRCP for diagnosing AIP is that the narrowed MPD seen on ERCP cannot be visualized on MRCP, because of the inferior resolution of MRCP compared with ERCP. Segmental narrowing of the MPD seen on ERCP was not visualized in $86 \%$ on MRCP, and distinguishing between AIP and PC was quite difficult on MRCP. However, in these cases, less upstream dilatation of the MPD on MRCP may suggest AIP rather than PC [26].

Extrapancreatic lesions occur in most cases of type I AIP and are positively correlated with serum IgG4 levels [27]. The biliary system, salivary glands, thyroid, lymph nodes, lung, retroperitoneum and kidney are most frequently involved [28]. Biliary and gallbladder involvement is often manifested as diffusive wall thickening [29]. In our case, CT image demonstrated enhancement and wall thickening of the gallbladder and common bile duct, which might be caused by obstruction due to the enlarged pancreas, or manifested as the extrapancreatic involvement in AIP.

\subsection{Steroid Treatment}

The dramatic steroid response is helpful for diagnosis of AIP, only when cancer 
is excluded. Steroid therapy brings about improvement in clinical symptoms and accompanying radiological and serological abnormalities, whose recommended starting dose is $0.6-1 \mathrm{mg} / \mathrm{kg} /$ day [5]. After $2-4$ weeks, the dose is tapered by 5 $\mathrm{mg}$ every $1-2$ weeks until it reaches $5-10 \mathrm{mg} /$ day [30]. Response to steroids is assessed in terms of pre- and poststeroid symptoms, liver biochemistry, serum IgG/IgG4 levels and radiological appearances. After confirmation of clinical remission, prednisolone is gradually reduced to the maintenance dose. Maintenance therapy with low-dose steroids is used to prevent disease relapse and maintain remission. Finally, steroids are usually completely discontinued.

Although, the relapse after remission of AIP is not uncommon. Relapses of AIP after remission with steroids usually occur in about one third of the patients [31]. Patients experience relapse of AIP, either during maintenance steroid therapy or after a complete discontinuation of steroids [32]. Relapse of AIP is often be retreated with steroids. The immunosuppressant drugs can also be used, especially to those patients, who is non-responsed to corticosteroids. In Yamabe's report, a 42-year-old man with AIP relapsed after the oral prednisolone treatment, and was retreated with methyl-prednisolone pulse therapy (500 $\mathrm{mg}$ /day) followed by oral prednisolone [33]. However, computed tomography, magnetic resonance imaging, and endoscopic ultrasound did not reveal sufficient improvement after 6 months of treatment. Therefore, an immunosuppressant (azathioprine) was introduced. Subsequent imaging analyses and endoscopic ultrasound fine needle aspiration revealed clear improvements in pathology.

Finally, our patient diagnosed of type I AIP underwent the steroid treatment lasting 3 months, who was gradually recovered. We present the case and take a review of literature in order to improve the early identification of AIP for the clinicians, especially for those gastroenterology and liver and ball surgical doctors.

\section{Conflict in the Publication}

The author has informed this patient who was reported in the paper. There is no conflict in the publication.

\section{References}

[1] Sarles, H., Sarles, J.C., Muratore, R. and Guien, C. (1961) Chronic Inflammatory Sclerosis of the Pancreas-An Autonomous Pancreatic Disease? The American Journal of Digestive Diseases, 6, 688-698. https://doi.org/10.1007/BF02232341

[2] Yoshida, K., Toki, F., Takeuchi, T., Watanabe, S., Shiratori, K. and Hayashi, N. (1995) Chronic Pancreatitis Caused by an Autoimmune Abnormality. Proposal of the Concept of Autoimmune Pancreatitis. Digestive Diseases and Sciences, 40, 1561-1568. https://doi.org/10.1007/BF02285209

[3] Members of the Criteria Committee for Autoimmune Pancreatitis of the Japan Pancreas Society (2002) Diagnostic Criteria for Autoimmune Pancreatitis by the Japan Pancreas Society. Journal of Japan Pancreas Society, 17, 585-587. (In Japanese) 
[4] Okazaki, K., Kawa, S., Kamisawa, T., et al. (2006) Clinical Diagnostic Criteria of Autoimmune Pancreatitis: Revised Proposal. Journal of Gastroenterology, 41, 626631. https://doi.org/10.1007/s00535-006-1868-0

[5] Shimosegawa, T., Chari, S.T., Frulloni, L., et al. (2011) International Consensus Diagnostic Criteria for Autoimmune Pancreatitis: Guidelines of the International Association of Pancreatology. Pancreas, 40, 352-358. https://doi.org/10.1097/MPA.0b013e3182142fd2

[6] Sadler, R., Chapman, R.W. and Simpson, D. (2011) The Diagnostic Significance of Serum IgG4 Levels in Patients with Autoimmune Pancreatitis: A UK Study. European Journal of Gastroenterology \& Hepatology, 23, 139-145. https://doi.org/10.1097/MEG.0b013e3283423486

[7] Paik, W.H., Ryu, J.K., Park, J.M., Song, B.J., Park, J.K., Kim, Y.T. and Lee, K. (2013) Clinical and Pathological Differences between Serum Immunoglobulin G4-Positive and -Negative Type 1 Autoimmune Pancreatitis. World Journal of Gastroenterology, 19, 4031-4038. https://doi.org/10.3748/wjg.v19.i25.4031

[8] Matsubayashi, H., Sawai, H., Kimura, H., Yamaguchi, Y., Tanaka, M., Kakushima, N., Takizawa, K., Kadooka, M., Takao, T., Hebbar, S., et al. (2011) Characteristics of Autoimmune Pancreatitis Based on Serum IgG4 Level. Digestive and Liver Disease, 43, 731-735. https://doi.org/10.1016/j.dld.2011.03.006

[9] Kamisawa, T., Chari, S.T., Lerch, M.M., Kim, M.H., Gress, T.M. and Shimosegawa, T. (2013) Recent Advances in Autoimmune Pancreatitis: Type 1 and Type 2. Gut, 62, 1373-1380. https://doi.org/10.1136/gutjnl-2012-304224

[10] Meng, Q., Xin, L., Liu, W., et al. (2015) Diagnosis and Treatment of Autoimmune Pancreatitis in China: A Systematic Review. PLOS ONE, 10, e0130466. https://doi.org/10.1371/journal.pone.0130466

[11] Kamisawa, T., Chari, S.T., Giday, S.A., et al. (2011) Clinical Profile of Autoimmune Pancreatitis and Its Histological Subtypes: An International Multicenter Survey. Pancreas, 40, 809-814. https://doi.org/10.1097/MPA.0b013e3182258a15

[12] Kamisawa, T. and Okamoto, A. (2008) IgG4-Related Sclerosing Disease. World Journal of Gastroenterology, 14, 3948-3955.

[13] Chari, S.T., Takahashi, N., Levy, M.J., et al. (2009) A Diagnostic Strategy to Distinguish Autoimmune Pancreatitis from Pancreatic Cancer. Clinical Gastroenterology and Hepatology, 7, 1097-1103. https://doi.org/10.1016/j.cgh.2009.04.020

[14] Okazaki, K., Kawa, S., Kamisawa, T., et al. (2014) Amendment of the Japanese Consensus Guidelines for Autoimmune Pancreatitis, 2013 I. Concept and Diagnosis of Autoimmune Pancreatitis. Journal of Gastroenterology, 49, 567-588. https://doi.org/10.1007/s00535-014-0942-2

[15] Kamisawa, T., Takuma, K., Egawa, N., Tsuruta, K. and Sasaki, T. (2010) Autoimmune Pancreatitis and IgG4-Related Sclerosing Disease. Nature Reviews Gastroenterology and Hepatology, 7, 401-409. https://doi.org/10.1038/nrgastro.2010.81

[16] Xin, L., He, Y.X., Zhu, X.F., et al. (2014) Diagnosis and Treatment of Autoimmune Pancreatitis: Experience with 100 Patients. Hepatobiliary \& Pancreatic Diseases International, 13, 642-648. https://doi.org/10.1016/S1499-3872(14)60263-0

[17] Hamano, H., Kawa, S., Horiuchi, A., Unno, H., Furuya, N., Akamatsu, T., et al. (2001) High Serum IgG4 Concentrations in Patients with Sclerosing Pancreatitis. The New England Journal of Medicine, 344, 732-738. https://doi.org/10.1056/NEJM200103083441005

[18] Chari, S.T., Smyrk, T.C., Levy, M.J., Topazian, M.D., Takahashi, N., Zhang, L., et al. (2006) Diagnosis of Autoimmune Pancreatitis: The Mayo Clinic Experience. Clini- 
cal Gastroenterology and Hepatology, 4, 1010-1016.

https://doi.org/10.1016/j.cgh.2006.05.017

[19] Levy, M.J., Wiesema, M.J. and Chari, S.T. (2006) Chronic Pancreatitis: Focal Pancreatitis or Cancer? Is There a Role for FNA/Biopsy? Autoimmune Pancreatitis. Endoscopy, 38, 30-35. https://doi.org/10.1055/s-2006-946648

[20] Chari, S.T., Kloeppel, G., Zhang, L., Notohara, K., Lerch, M.M. and Shimosegawa, T. (2010) Histopathologic and Clinical Subtypes of Autoimmune Pancreatitis: The Honolulu Consensus Document. Pancreas, 39, 549-554. https://doi.org/10.1097/MPA.0b013e3181e4d9e5

[21] Deshpande, V., Gupta, R., Sainani, N., Sahani, D.V., Virk, R., Ferrone, C., Khosroshahi, A., Stone, J.H. and Lauwers, G.Y. (2011) Subclassification of Autoimmune Pancreatitis: A Histologic Classification with Clinical Significance. The American Journal of Surgical Pathology, 35, 26-35. https://doi.org/10.1097/PAS.0b013e3182027717

[22] Notohara, K., Burgart, L.J., Yadav, D., Chari, S. and Smyrk, T.C. (2003) Idiopathic Chronic Pancreatitis with Periductal Lymphoplasmacytic Infiltration: Clinicopathologic Features of 35 Cases. The American Journal of Surgical Pathology, 27, 1119-1127. https://doi.org/10.1097/00000478-200308000-00009

[23] Gompertz, M., Morales, C., Aldana, H., Castillo, J. and Bergera, Z. (2015) Cystic Lesions in Autoimmune Pancreatitis. Case Reports in Gastroenterolog, 9, 366-374. https://doi.org/10.1159/000441998

[24] Irie, H., Honda, H., Baba, S., et al. (1998) Autoimmune Pancreatitis: CT and MR Characteristics. American Journal of Roentgenology, 170, 1323-1327.

https://doi.org/10.2214/ajr.170.5.9574610

[25] Sugiyama, Y., Fujinaga, Y., Kadoya, M., Ueda, K., Kurozumi, M., Hamano, H., et al. (2012) Characteristic Magnetic Resonance Features of Focal Autoimmune Pancreatitis Useful for Differentiation from Pancreatic Cancer. Japanese Journal of Radiology, 30, 296-309. https://doi.org/10.1007/s11604-011-0047-2

[26] Kamisawa, T., Tu, Y., Egawa, N., et al. (2009) Can MRCP Replace ERCP for the Diagnosis of Autoimmune Pancreatitis? Abdominal Imaging, 34, 381-384. https://doi.org/10.1007/s00261-008-9401-y

[27] Naitoh, I., Nakazawa, T., Ohara, H., Ando, T., Hayashi, K., Tanaka, H., Okumura, F., Miyabe, K., Yoshida, M., Sano, H., et al. (2010) Clinical Significance of Extrapancreatic Lesions in Autoimmune Pancreatitis. Pancreas, 39, e1-e5. https://doi.org/10.1097/MPA.0b013e3181bd64a1

[28] Milosavljevic, T., Kostic-Milosavljevic, M., Jovanovic, I. and Krstic, M. (2012) Extraintestinal Manifestations of Autoimmune Pancreatitis. Digestive Diseases, 30, 220-223. https://doi.org/10.1159/000336708

[29] Nishino, T., Toki, F., Oyama, H., Oi, I., Kobayashi, M., Takasaki, K. and Shiratori, K. (2005) Biliary Tract Involvement in Autoimmune Pancreatitis. Pancreas, 30, 76 82.

[30] Kamisawa, T., Okazaki, K., Kawa, S., Shimosegawa, T. and Tanaka, M. (2010) Japanese Consensus Guidelines for Management of Autoimmune Pancreatitis: III. Treatment and Prognosis of AIP. Journal of Gastroenterology, 45, 471-477. https://doi.org/10.1007/s00535-010-0221-9

[31] Chari, S.T. (2007) Current Concepts in the Treatment of Autoimmune Pancreatitis. JOP, 8, 1-3.

[32] Wakabayashi, T., Kawaura, Y., Satomura, Y., Watanabe, H., Motoo, Y. and Sawabu, N. (2005) Long-Term Prognosis of Duct-Narrowing Chronic Pancreatitis: Strategy for Steroid Treatment. Pancreas, 30, 31-39. 
[33] Yamabe, A., Irisawa, A., Notohara, K., Shibukawa, G., Fujisawa, M., Sato, A., Yoshida, Y., Arakawa, N., Ikeda, T., Igarashi, R., Maki, T. and Yamamoto, S. (2016) A Case of Autoimmune Pancreatitis Effectively Treated with an Immunosuppressant (Azathioprine). Clinical Journal of Gastroenterology, 9, 324-328.

https://doi.org/10.1007/s12328-016-0673-4

Submit or recommend next manuscript to OALib Journal and we will provide best service for you:

- Publication frequency: Monthly

- 9 subject areas of science, technology and medicine

- Fair and rigorous peer-review system

- Fast publication process

- Article promotion in various social networking sites (LinkedIn, Facebook, Twitter, etc.)

- Maximum dissemination of your research work

Submit Your Paper Online: Click Here to Submit

Or Contact service@oalib.com 